\title{
Unprecedented High-Modulus High-Strength Tapes and Films of Ultrahigh Molecular Weight Polyethylene via Solvent-Free Route
}

\author{
Sanjay Rastogi, ${ }^{*,+, \perp}$ Yefeng Yao, ${ }^{\S}$ Sara Ronca, ${ }^{\ddagger}$ Johan Bos, ${ }^{\perp}$ and Joris van der Eem ${ }^{\perp}$ \\ ${ }^{\dagger}$ Department of Chemistry and Chemical Engineering, Eindhoven University of Technology, Eindhoven, The Netherlands \\ ${ }^{\ddagger}$ Department of Materials, Loughborough University, Leicestershire LE11 3TU, U.K. \\ ${ }^{\S}$ Physics Department \& Shanghai Key Laboratory of Magnetic Resonance, East China Normal University, Shanghai, 200062, P. R. China \\ ${ }^{\perp}$ Research Institute, Teijin Aramid B.V., Velperweg 76, Arnhem, The Netherlands
}

Supporting Information

\section{INTRODUCTION}

The ease in processability of synthetic polymers has led to their fast growth as commodity as well as engineering plastic, whereas the latter are used in case of demanding applications. Among several, the most well-studied polymer is probably polyethylene. This polymer, based on a simple $-\left(\mathrm{CH}_{2}-\mathrm{CH}_{2}\right)-$ repeating unit, can be used for day-to-day applications as well as for more challenging duties such as prostheses, soft and hard ballistics, light weighted ropes, etc. The wide range of physical and mechanical properties is accessible due to fine control of the molecular architecture, coming from the better understanding of polymer synthesis. The development of a material with the desired properties requires the combination of several disciplines in science, including chemistry, physics, rheology, and processing. It is recognized that, for linear polyethylene, virtually free of chain branching, the physical properties such as wear, abrasion resistance, and impact strength enhance with increasing molar mass. However, the material becomes more difficult to process because the zero shear viscosity follows the well-known power law $\eta_{0} \propto M^{3.4}$, thus making the polymer having weight-average molar mass greater than 1 million $\mathrm{g} / \mathrm{mol}$ nearly impossible to process via the conventional processing route. ${ }^{1,2}$ The cause for the increase in viscosity, when increasing the molar mass of the polymer, is related to the increasing number of physical entanglement (friction points due to contact of chain segments) as the molar mass between the entanglement, $M_{\mathrm{e}}$, is considered to be a constant for a given polymer. For example, in the case of linear polyethylene, the molar mass between entanglement is $\sim 1200 \mathrm{~g} / \mathrm{mol}^{3}$

The number of entanglement per chain can be effectively suppressed by dissolution of the polymer in a suitable solvent. In dilute solutions, below the so-called overlap concentration $\Phi^{*}$, the number of entanglement per chain can be reduced considerably. ${ }^{4}$ In the case of crystallizable polymers, such as linear polyethylene, the reduced entanglement density in the solution can be made permanent since the long chain molecules form folded-chain crystals on cooling where entanglement reside in the amorphous phase, a well-studied phenomenon in polymer physics. ${ }^{5,6}$ The reduction of entanglement in the amorphous region of the semicrystalline polymer favor the postdrawing process. ${ }^{7}$ Following these concepts, the existing commercial route has been adopted to develop high-modulus high-strength fibers, where dissolution of less than 6 wt \% of the ultrahigh molecular weight polymer in a high boiling point solvent such as decalin ${ }^{8}$ is required. Prior to removal of the 94 wt \% of the solvent, the solution is cooled for crystallization. The disentangled solid state thus achieved is used for its ease in drawability to make high-modulus high-strength fibers. However, on heating the solution crystallized polymer, the disentangled chains tend to re-entangle, erasing the disentangled state. ${ }^{9,10}$

A more elegant and also technologically more advanced way to generate disentangled linear polyethylene crystals is via direct polymerization in the reactor. ${ }^{11-13}$ At low polymerization temperatures and low catalyst activity/concentration, individual growing chains will form their own folded chain crystals. In the limiting case where the growing chains are separated far enough from each other, ultimately monomolecular crystals can be formed. If completely disentangled PE structures can be obtained via solution-crystallization and/or via direct controlled synthesis, the intriguing question is whether this disentangled state will be preserved upon melting and what is the time scale to generate a fully entangled equilibrium polymer melt. Some of these fundamental questions have been addressed previously. ${ }^{14-18}$

The deformation behavior in the solid-state processing (free of solvent), either uniaxial or biaxial, is likely to be strongly dependent on the topological constraints that are established in the amorphous region of the polymer. Thus, in this publication with the help of solid-state NMR studies we have followed differences between the amorphous regions of the nascent entangled and the nascent disentangled polyethylenes synthesized via the conventional Ziegler-Natta $(\mathrm{Z}-\mathrm{N})$ and the direct controlled synthesis, respectively. Uniaxial and biaxial deformation aspects of the disentangled polyethylene have been compared with the entangled polyethylene. Resultant properties of the two materials against the draw ratio have been investigated. Crystallinity measurements and the resultant tensile properties in the drawn tapes have been investigated by solid-state NMR. With the help of X-ray diffraction studies, the cause of the high modulus in the solid-state processed materials has been compared with the polyethylene processed via the solution route.

Received: March 23, 2011

Revised: June 6, 2011 
In the conventional synthesis of the UHMWPE heterogeneous $\mathrm{Z}-\mathrm{N}$ catalysts are used, mainly in the slurry phase, where the catalyst is dispersed in a solvent and the polymerization conditions such as temperature, pressure, and catalyst concentration are tuned for maximum yield. ${ }^{19}$ The polymer thus obtained from the spatially close catalytic sites normally possesses a broad molar mass distribution greater than 6 , and it is in the equilibrium entangled state. Since the discovery of homogeneous, single-site catalysts based on organometallic complexes of group IV metals, it has been possible to achieve a finer control over the molecular architecture of the polymer produced. $^{20}$ For polymerization purposes the homogeneous catalyst is dissolved in a suitable solvent (usually toluene) and activated with a cocatalyst (usually an alkylaluminum species), prior to its transfer in the polymerization reactor containing the (same) solvent saturated with ethylene at the desired partial pressure. ${ }^{21}$ A very desirable feature of this class of catalyst is the uniformity of the active sites that result in a narrow molecular weight distribution of the obtained polymer (typically around 2 ), in contrast with the broad distribution typical of heterogeneous catalysts. When the polymerization rate of such a catalyst is considerably higher than the rate of any termination process, it is possible to achieve polymers of high molecular weight: in some particular cases, the incidence of termination processes can be so low that the polymerization is considered to be "living" in the time scale of the experiment. A number of catalysts having such characteristics have appeared in the literature: for example, since 2000, Fujita and co-workers ${ }^{22}$ have extensively published on a series of complexes based on group IV metals bearing bis(phenoxyimine) type ligands (generally indicated as FI catalysts). These complexes, when activated with a suitable cocatalyst (the most common of which is methylaluminoxane, $\mathrm{MAO}$ ), can produce a variety of polyolefin architectures depending on the metal/ligand pair chosen. In particular, the complex depicted in the inset of Figure $1 \mathrm{a},{ }^{23}$ when activated with $\mathrm{MAO}$, can produce polyethylene of number-average molar mass $M_{\mathrm{n}} 412 \times 10^{3} \mathrm{~g} / \mathrm{mol}$ with high activity in very mild conditions ( 1 bar partial pressure of ethylene and room temperature) in as low as $1 \mathrm{~min}$ reaction. According to Fujita et al., this catalytic system is also living for at least $30 \mathrm{~min}$ in the same conditions. These characteristics make it an ideal candidate for the synthesis of disentangled UHMWPE directly from polymerization. The main conditions required to reduce the number of entanglements during the reaction itself are (i) low polymerization temperature, so that the crystallization rate is faster than the polymerization rate, and (ii) low concentrations of active sites, to minimize the interaction of the growing chains.

In this Communication we address mechanical properties of the disentangled polyethylene, synthesized using the catalytic system, and processed below its melting point both along the uniaxial and the biaxial directions to make tapes and films.

\section{EXPERIMENTAL METHODS}

Materials. All manipulations of air- and moisture-sensitive compounds were performed under a nitrogen or argon atmosphere using standard high-vacuum Schlenk techniques or in a glovebox. Toluene (99.8\%, anhydrous) and methylaluminoxane (MAO, $10 \mathrm{wt} \%$ solution in toluene) were purchased from Aldrich, ethylene (grade 3.0) was purchased from BOC, and FI precatalyst (reported in Figure 1) was purchased from
MCat. All reagents were used as received. Commercial samples of (entangled) ultrahigh molecular weight polyethylene were synthesized using $\mathrm{Z}-\mathrm{N}$ catalyst. Solution spun fibers are obtained from the commercial source, Dyneema SK75.

Synthesis of Disentangled UHMWPE. A wall-mounted, $10 \mathrm{~L}$ jacketed Pyrex reactor was equipped with a double plane propeller blade mechanical stirrer, a temperature probe, a gas inlet/ outlet, and a rubber septum for catalyst injection. The reactor was kept overnight at $125^{\circ} \mathrm{C}$, and then the temperature was brought to room temperature while the vessel was purged with three cycles of vacuum/nitrogen. The required amount of toluene ( $5 \mathrm{~L}$ ) was transferred under a nitrogen gas stream into the vessel, and the temperature was set to the desired value $\left(10^{\circ} \mathrm{C}\right)$ by means of a thermostat. When the desired temperature was reached, the stream of gas was switched from nitrogen to ethylene. Ethylene uptake was controlled by means of a Buchi press flow gas controller bpc 6002. When the solution was saturated with ethylene at the desired partial pressure ( 1 bar ethylene), MAO (50 mL) was added, followed by a solution of the catalyst $(50 \mathrm{mg})$ in toluene $+\mathrm{MAO}$ to start the polymerization. The reaction was carried on for the required time $(1 \mathrm{~h})$ under vigorous stirring and constant feed of ethylene and then quenched by addition of acidified methanol $\left(\mathrm{CH}_{3} \mathrm{OH} / \mathrm{HCl}\right.$ $95 / 5 \mathrm{v} / \mathrm{v}$ ). The polymer was filtered out, washed with additional methanol, and oven-dried under vacuum at $40{ }^{\circ} \mathrm{C}$ for one night.

Scanning Electron Micrographs. SEM investigations on morphologies of nascent reactor powders were carried out with a high-resolution FEG SEM (Carl Zeiss Leo 1530 VP) operated at $5 \mathrm{kV}$. As-polymerized particles were carefully deposited on SEM stabs, and the samples were coated with gold by a sputtering technique.

Polymer Processing of Nascent Disentangled and Entangled Powders. A general procedure for the preparation of tapes is as follows: $25 \mathrm{~g}$ of polymer powder is poured into a mold with a cavity of $620 \mathrm{~mm}$ in length and $30 \mathrm{~mm}$ in width and compression-molded at $130 \mathrm{bar}$ for $10 \mathrm{~min}$ to form a sheet. The sheet is preheated for at least $1 \mathrm{~min}$ and rolled with a Collin calander (diameter rolls: $250 \mathrm{~mm}$, slit distance $0.15 \mathrm{~mm}$, inlet speed $0.5 \mathrm{~m} / \mathrm{min}$ ). The tape is immediately stretched on a roll (speed $2.5 \mathrm{~m} / \mathrm{min}$ ). The rolled and stretched tape is further stretched in two steps on a $50 \mathrm{~cm}$ long oil heated hot plate. The tape comes in contact with the hot plate after $20 \mathrm{~cm}$ from the entrance of the hot plate. The draw ratio is obtained by dividing specific weight of the sheet prior to deformation by the specific weight of the tape after stretching. Typical processing temperatures of entangled PE (ePE) and disentangled PE (dPE) are given in Table 1.

Tensile Testing. Tensile properties are measured using an Instron 5566 tensile tester at room temperature $\left(25^{\circ} \mathrm{C}\right)$. To avoid any slippage, the side action grip clamps with flat jaw faces are used. The nominal gauge length of the specimen is $100 \mathrm{~mm}$, and the test is performed at a constant rate of extension (crosshead travel rate) $50 \mathrm{~mm} / \mathrm{min}$. The breaking tenacity (or tensile strength) and modulus (segment between 0.3 and $0.4 \mathrm{~N} /$ tex) are determined from the force against displacement between the jaws.

X-ray Diffraction Studies. For the measurements a Bruker D8 diffractometer in $\theta / 2 \theta$ geometry is employed. The D8 is equipped with parallel beam optics, scintillation detector, and autochanger. The optics consists of a primary $60 \mathrm{~mm}$ Göbel focusing mirror (a parabolic Ni/C multilayer device) providing $\mathrm{Cu} \mathrm{K} \alpha$ radiation $(\mathrm{K} \alpha$ wavelength $=1.5418 \AA$ Á $)$ and $0.12^{\circ}$ Soller 
(a)

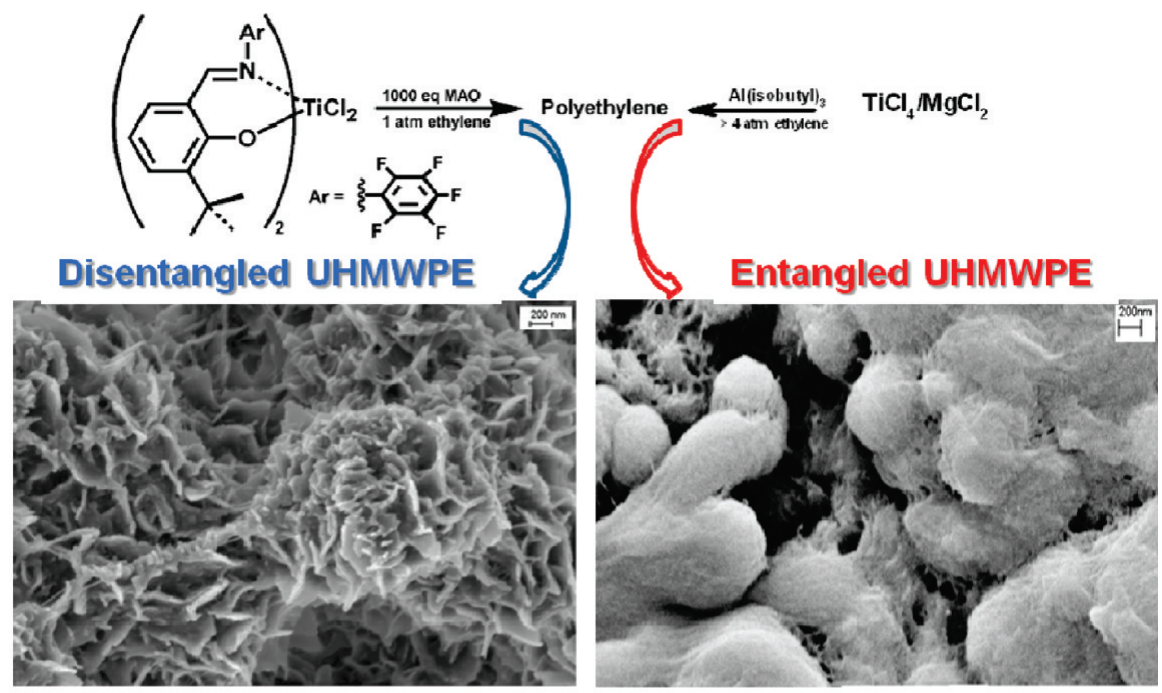

(b)

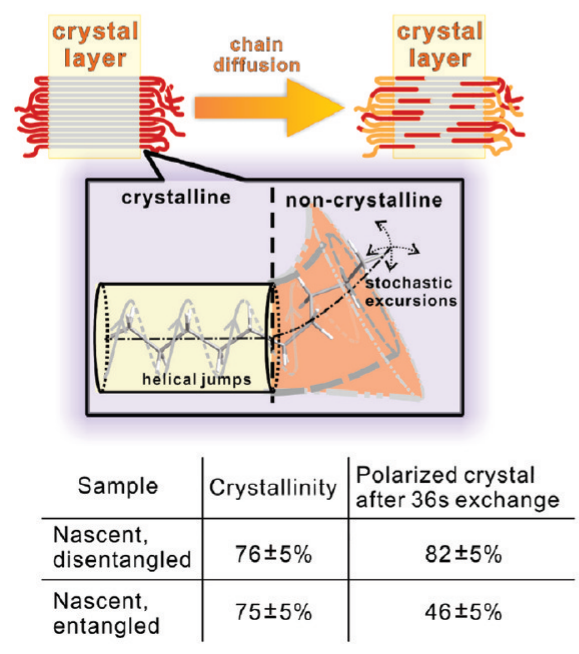

Nascent disentangled Nascent entangled

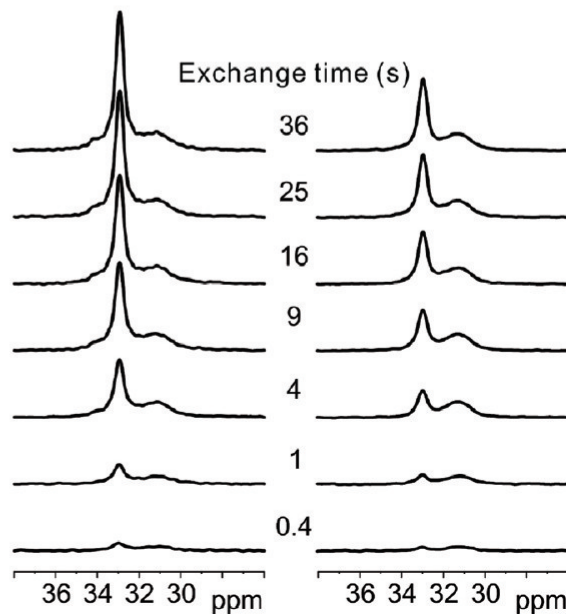

Figure 1. Morphological and molecular distinction in the nascent UHMWPE polymers, as obtained after synthesis. (a) A comparison of scanning electron micrographs obtained on UHMWPE samples synthesized using homogeneous (left) and heterogeneous (right) catalysts. (b) Morphological differences depicted in (a) can be realized at the segmental level by following the diffusion of methylene units of linear polyethylene from amorphous to crystalline regions. Such a possibility is realized by making judicious choice of pulse sequences in solid-state NMR studies. The cartoon depicts excursion of methylene sequences from amorphous to crystalline region. Exchange process as a function of exchange time has been measured in the disentangled and entangled samples of UHMWPE. The table provides a snapshot in the polarization of crystal due to motion of methylene sequences from amorphous to crystalline region after $36 \mathrm{~s}$. The differences in polarization are attributed to the distinction in the two morphologies of the nascent powders of UHMWPE.

slits. Generator setting was kept at $40 \mathrm{kV}$ and $35 \mathrm{~mA}$. The specimen (tape, film, or sheet) is mounted on a sample holder with double-sided mounting tape. The standard D8 sample holder is subsequently placed into the D8 diffractometer in reflection geometry (with normal of the tape perpendicular to the goniometer and the sample holder). The scan range for the diffraction pattern is from $5^{\circ}$ to $40^{\circ}(2 \theta)$ with a step size of $0.02^{\circ}$ $(2 \theta)$ and a counting time of $2 \mathrm{~s}$ per step. The sample holder spins during the measurement with $15 \mathrm{rpm}$ around the normal of the tape. For determination of the crystal plane orientation the peak area of the 110 and 200 reflections is determined by a standard profile fitting routine. The crystal plane orientation (CPO) parameter is defined as the ratio between the determined 200 and 110 peak areas. For the measurements carried out in the reflection geometry, the ratio between the 200 and 110 peak areas for a specimen with randomly oriented crystallites is around 0.3 , but for solid state drawn polyethylene tape samples the 200 planes are preferentially oriented parallel to the film surface, resulting in a higher value of the 200/110 peak area ratio. Orientation in the fiber filaments is measured by mounting the filament on goniometer in transmission mode.

Dynamic NMR. All solid-state NMR studies are performed on a Bruker DSX spectrometer operating at $500 \mathrm{MHz}{ }^{1} \mathrm{H}$ Lamor frequency. Advanced NMR recoupling techniques correlating isotropic chemical shifts under magic angle spinning (MAS) with motionally averaged ${ }^{1} \mathrm{H}-{ }^{13} \mathrm{C}$ heteronuclear dipole-dipole couplings are used to study the local chain dynamics in the samples. The $2.5 \mathrm{~mm}$ MAS double resonance probe is used to 
Table 1. Processing Temperatures and Solid-State Extrusion of UHMWPE

\begin{tabular}{llll}
\multicolumn{1}{c}{ operation } & processing parameter & dPE & ePE \\
compression molding & $T$ mold, ${ }^{\circ} \mathrm{C}$ & 129 & 136 \\
rolling & $T$ preheated, ${ }^{\circ} \mathrm{C}$ & 125 & 138 \\
& $T$ calander, ${ }^{\circ} \mathrm{C}$ & 130 & 145 \\
\multirow{2}{*}{ stretching } & $T$ hot plate, ${ }^{\circ} \mathrm{C}$ & 139 & 154 \\
\hline
\end{tabular}

study the chain diffusion between noncrystalline and crystalline regions based on ${ }^{13} \mathrm{C}$ exchange type experiment under MAS at $6 \mathrm{kHz}$. The $90^{\circ}$ pulse length in the $2.5 \mathrm{~mm}$ MAS is adjusted to $2.5 \mu$ s on both channels. All experiments are performed at $50^{\circ} \mathrm{C}$.

\section{- RESULTS AND DISCUSSION}

Because of the living polymerization behavior of the chosen catalytic system, it has been possible to synthesize polymers of different weight-average molar masses ranging from 2 million to 9 million $\mathrm{g} / \mathrm{mol}$. For the studies performed in this publication, the disentangled polyethylene having a number-average molar mass greater than 1 million $\mathrm{g} / \mathrm{mol}$ and weight-average molar mass around 2.8 million $\mathrm{g} / \mathrm{mol}$ has been used. The molar mass and molar mass distributions have been determined via rheological studies, according to the method reported elsewhere. ${ }^{24}$

Following the method described from Klimke, ${ }^{25}$ the absence of chain branching up to $10^{4}$ carbon atoms is confirmed. These findings are along the anticipated absence of chain branching in polyethylenes synthesized using the FI catalyst.

A typical morphology obtained following the homogeneous synthesis route as viewed by scanning electron microscopy on the nascent polymer obtained after polymerization is shown in Figure 1a. The aggregate platelets having $20 \mathrm{~nm}$ thickness and surface area of several micrometers are in accordance with the dimensions for the single crystals of linear polyethylene. Considering the weight-average molar mass and molar mass distribution of this sample, $\sim 2.8$ million $\mathrm{g} / \mathrm{mol}$ and 3.0 , respectively, it is fascinating to anticipate that a chain comprising $200000 \mathrm{CH}_{2}$ units on average, having an end-to-end stretched chain distance of $\sim 25000 \mathrm{~nm}$, tends to fold back and forth 1250 times within the crystal thickness of $20 \mathrm{~nm}$. Morphology of commercial sample (entangled), obtained with heterogeneous $\mathrm{Z}-\mathrm{N}$ catalyst, does not show the presence of single platelets as observed in the sample obtained with the homogeneous catalyst. Distinct differences in the two morphologies imply loose packing of platelike crystals, having an average thickness of $\sim 20 \mathrm{~nm}$, in the powder obtained from homogeneous synthesis compared to the dense aggregate of crystals seen in the sample synthesized using the heterogeneous catalyst. Platelets of crystals obtained from homogeneous catalyst could be compressed and drawn in the solid state, in both the uniaxial and biaxial direction, in a broad temperature window ranging between 120 and $145{ }^{\circ} \mathrm{C}$. The dense polymer obtained from heterogeneous catalyst could be drawn in a narrow temperature window of $2-3^{\circ} \mathrm{C}$ near $142{ }^{\circ} \mathrm{C}$, along uniaxial direction only. The unique morphology of the polymer obtained from the homogeneous catalytic system is suggestive of loose packing of single crystals in disentangled UHMWPE.

Distinction in the morphologies of the disentangled and entangled at the molecular level could be probed with the help of dynamic solid-state NMR (Figure 1b). Such studies performed on the nascent crystals of both disentangled and entangled
UHMWPE, where the relaxation processes in the amorphous and the crystalline regions could be decoupled by making judicious choice of the pulse sequences, show fast exchange process of segments from amorphous to crystalline regions. The difference between the two samples lies in the rate of exchange process of methylene units from amorphous to crystalline state. For an example after $36 \mathrm{~s}$, the crystal of the disentangled UHMWPE gains polarization by $82 \%$ compared to $46 \%$ polarization gained by the entangled UHMWPE, thus suggesting faster exchange process in the disentangled sample compared to the entangled sample. These observations are along the lines of experiments performed on the solution crystallized UHMWPE where the disentangled state is achieved by dissolution of less than $2 \mathrm{wt} \%$ of UHMWPE in $98 \mathrm{wt} \%$ of decalin. ${ }^{26}$

Thermal scans of both nascent polymers, disentangled and entangled, during first heating show a melting peak at $142{ }^{\circ} \mathrm{C}$, whereas on second heating the two samples now crystallized from melt show a decrease in the melting temperature by $6{ }^{\circ} \mathrm{C}$. Considering the similarity in the crystal thicknesses of the nascent and the melt crystallized samples, both $\sim 20 \mathrm{~nm}$ along the chain direction, the difference in the melting temperatures cannot be simply explained by the Gibbs-Thomson equation which takes into consideration crystal thickness only. The difference in the melting temperatures is explained by invoking the melting kinetics that differs between the entangled and the disentangled nascent polymers; for details see refs 16 and 18 . The restricted chain motion in the amorphous region of the nascent polymer causes an increase in the apparent chain length, greater than the crystal thickness that needs to adopt the random coil state on melting. These findings are further strengthened on considering differences in the chain mobility of the amorphous regions of the two nascent samples and melt-crystallized samples as evident from the solid-state NMR studies. ${ }^{27}$

Rheological studies performed on the entangled and disentangled nascent polymer are shown in Figure 4 of ref 18. To recall, the increase in the storage modulus of the disentangled nascent polymer melt suggests entanglements formation with time. On the contrary, no significant increase in modulus is observed on melting of the entangled polymer independent of the rate at which the polymer is heated from solid to melt state.

The combined NMR, DSC, and rheological studies confirm the disentangled nature of the samples synthesized using homogeneous catalyst, thus invoking the possibility of the solid-state, solvent-free, processing along the uniaxial and biaxial directions. Such a possibility was already anticipated by Kanamoto and Porter. ${ }^{28-31}$ These authors made several attempts to process polyethylene samples synthesized under controlled conditions. However, due to the restricted processing temperature window of $2-3^{\circ} \mathrm{C}$ in the vicinity of melting point and the presence of air voids in the compressed films, the modulus (107 GPa) and strength (1.4 GPa) of the tapes obtained by Kanamoto and Porter for the maximum draw ratio of 77 times were much lower than those reported for the fibers obtained via the solution spinning route. Rotzinger, Chanzy, and Smith ${ }^{32}$ followed the concepts of controlled synthesis where polymerization temperature was maintained at $-40{ }^{\circ} \mathrm{C}$ and ethylene pressure below atmospheric pressure. Catalyst used for the synthesis was a $\mathrm{Z}-\mathrm{N}$ type catalyst based on vanadium. The polymer produced by this synthesis route was limited in amount, and thus the reported experimental findings were limited. However, the potential to achieve mechanical properties equivalent to solution-spun route could be shown. Because of difficulties in synthesis and 
processing, the solid-state processing route was not pursued further, and for more than a decade it has been a subject of some academic interest only.

The synthesis route applied in this study is able to provide control over the molecular weight and molecular weight distribution while maintaining the high activity of the catalyst. Such a possibility leads to the synthesis of sufficiently large amount of the polymer to perform a series of experiments. For solid-state processing of the disentangled polyethylene below its equilibrium melting point, the powder is compressed at $120^{\circ} \mathrm{C}$ into a strip of $0.2 \mathrm{~cm}$ thickness and $70 \mathrm{~cm}$ length. After compression, the density of the strip is measured to be $0.98 \mathrm{~g} / \mathrm{cm}^{3}$. The compressed strip is rolled and stretched at $130{ }^{\circ} \mathrm{C}$ by nearly 4 times. The compressed, rolled, and stretched film is subjected to three consecutive stretches at 135,140 , and $145{ }^{\circ} \mathrm{C}$ by draw ratios of 20,60, and 180, thus leading to an ultimate draw ratio of more than 180 . To avoid any influence of slippage in determination of the draw ratio during solid-state deformation, the quoted draw ratios are measured as mass/length before and after deformation. It should be realized that the melting temperature of the constrained oriented tapes having extended chains increases from 142 to $150^{\circ} \mathrm{C}$. Thus, all the drawing steps have been performed below the melting temperature in a broad temperature window of more than $20^{\circ} \mathrm{C}$. At each drawing step the tensile strength and modulus of the tapes are measured. Gradual increase in the modulus and strength at each drawing step is observed. The variation in modulus and strength with drawing ratio has been depicted in Figure 2. The draw ratio is determined by measuring mass/length of the sample prior and after the deformation. The appearance of neck formation during deformation and the near absence of strain hardening confirm the disentangled nature of the crystals of polyethylene obtained with the homogeneous catalyst. To avoid complexity arising due to air voids between fibrils in tapes, modulus and tensile strength are measured in $\mathrm{N} /$ tex.

On the contrary to the disentangled UHMWPE synthesized using the homogeneous catalyst, the entangled sample (synthesized in laboratory scale or obtained commercially) can be processed only in a narrow temperature window of $2-3{ }^{\circ} \mathrm{C}$ in the vicinity of melting temperature. Figure 2 shows that the entangled sample has similar modulus but distinctly lower tensile strength compared to the disentangled polymer. However, with advances made in polymer synthesis and control over drawing temperature, compared to the earlier studies, ${ }^{31}$ it has been now possible to achieve higher tensile strength and modulus in the entangled commercial samples. The experiments on some of the commercial samples have been performed in our laboratories, and details are provided in ref 33 .

Films having bulk density of $0.98 \mathrm{~g} / \mathrm{cm}^{3}$ obtained after compression of the nascent disentangled and entangled samples were stretched on a preheated hot plate. From Figure 2 it is apparent that tensile strength to break, i.e. tenacity, increases with stretching of the polymers. For the same draw ratio, as measured by change in mass with deformation, tenacity of the disentangled sample is considerably higher than the entangled sample. For the draw ratio of nearly 200, maximum tenacity achieved in the entangled sample is $2.3 \mathrm{~N}$ /tex compared to 3.8 $\mathrm{N} /$ tex for the disentangled sample. For the maximum draw ratio of 200, modulus of the disentangled polymer is closer to $200 \mathrm{~N} /$ tex compared to $150 \mathrm{~N} /$ tex for the entangled sample. The maximum tensile strength and modulus of the solution-spun fibers are 3.5 and $135 \mathrm{~N} /$ tex, respectively, as measured on
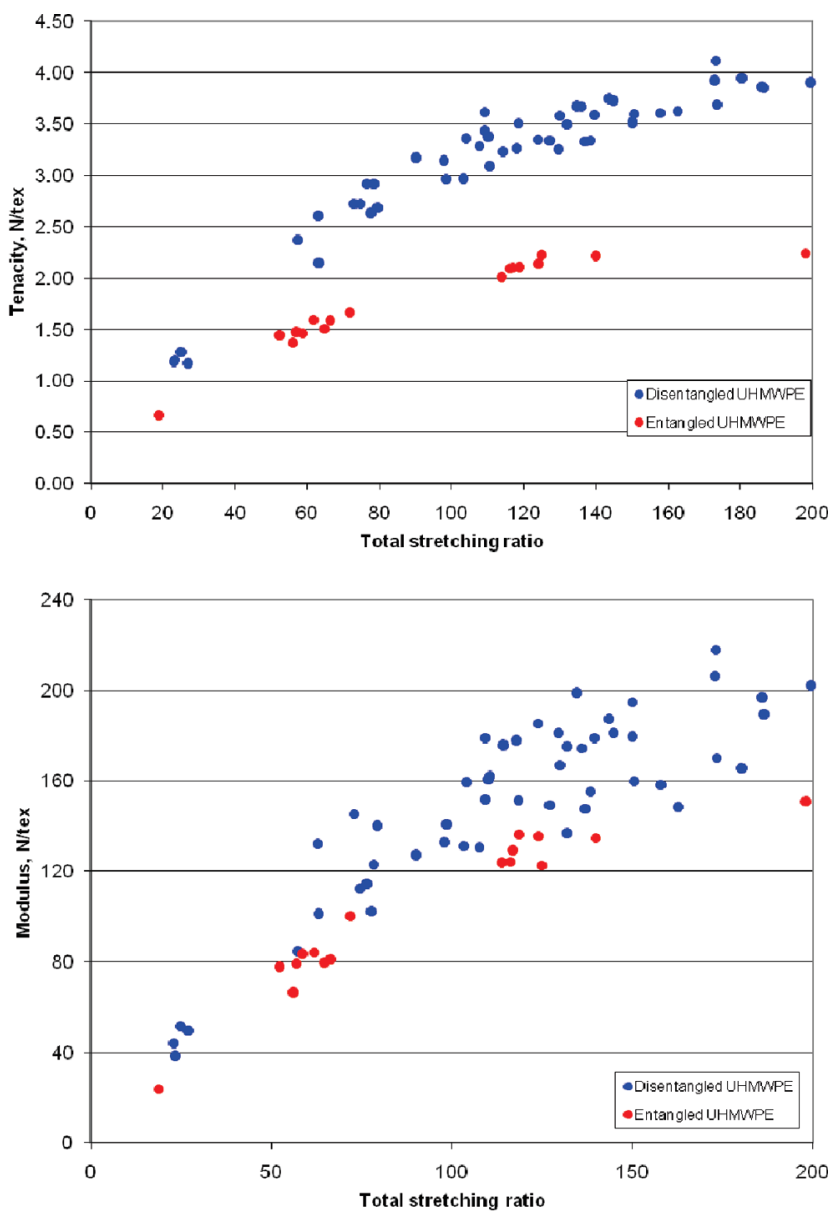

Increasing stretch ratio

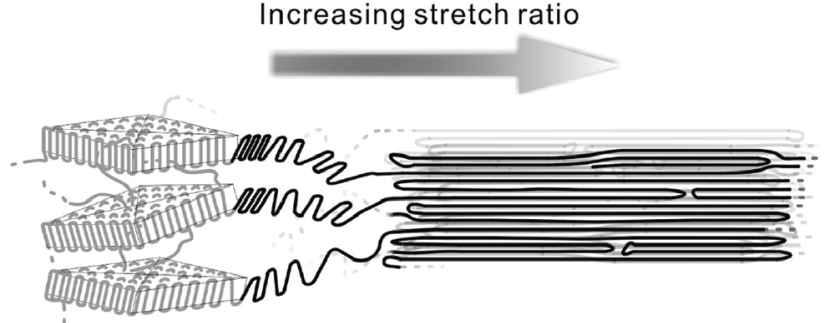

Figure 2. Increase in tensile strength (tenacity) and modulus with increasing draw ratio. Figure below shows variation in modulus with increasing draw ratio. For the bulk density of $0.98 \mathrm{~g} / \mathrm{cm}^{3}, 1.0 \mathrm{~N} / \mathrm{tex}=$ $0.98 \mathrm{GPa}$.

Dyneema SK75 at $25{ }^{\circ} \mathrm{C}$ in our laboratory (see Supporting Information), whereas the reported values in the literature for solution spinning of $1 \mathrm{wt} \%$ of the polymer, in $99 \mathrm{wt} \%$ of the solvent, are 120 and $3.2 \mathrm{GPa}$ for tensile modulus and tensile strength, respectively. ${ }^{34}$ It is to be noted that in solution spinning modulus, strength, and draw ratio are strongly dependent on polymer concentration in solution and weight- and numberaverage molar mass of the polymer. ${ }^{4,7}$ On the contrary, the controlled synthesis route adopted for solid-state processing reduces the entanglement density with increasing molar mass, thus making processability of the disentangled ultrahigh molar mass polymer easier. ${ }^{35}$

The described method for determining modulus and strength of tapes is also applied to solution-spun high grade fiber, commercially available in the market. For our purpose we have 

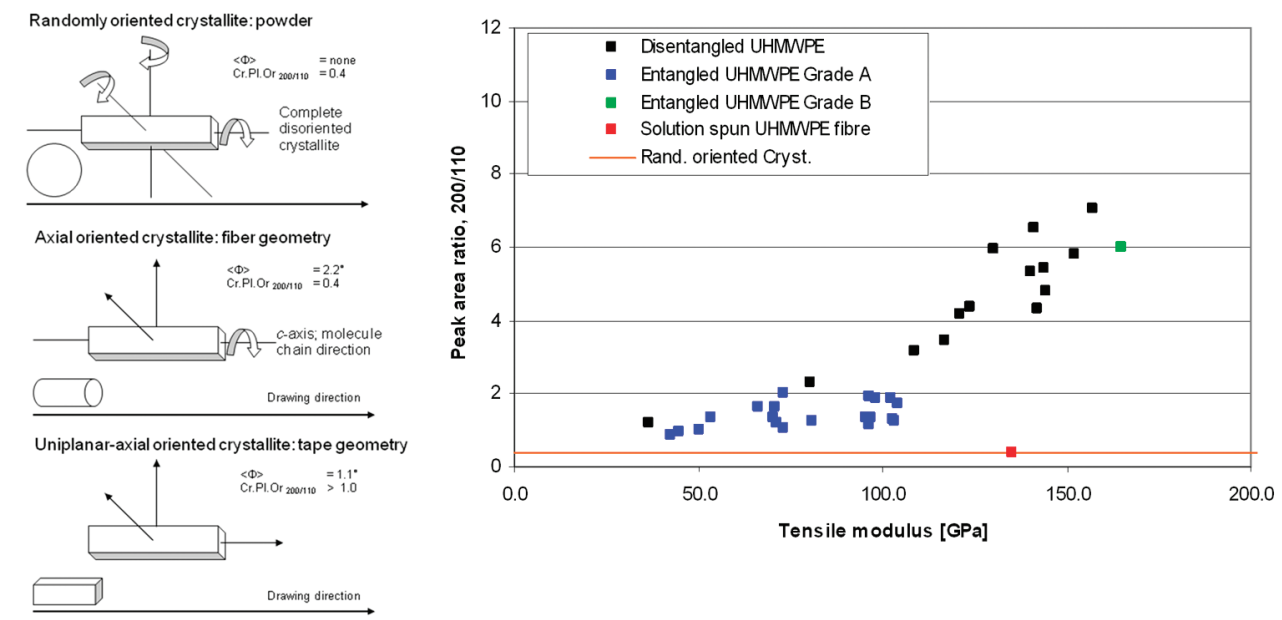

\begin{tabular}{|l|c|c|c|c|}
\hline & $\begin{array}{c}\text { Tensile Strength } \\
{[\mathrm{GPa}]}\end{array}$ & $\begin{array}{c}\text { Modulus } \\
{[\mathrm{GPa}]}\end{array}$ & $\begin{array}{c}\left\langle\Phi_{\phi \theta}\right\rangle \\
{\left[{ }^{\circ}\right]}\end{array}$ & $\begin{array}{c}\text { C.P.O. } \\
\text { peak ratio }\end{array}$ \\
\hline Disentangled UHMWPE & 3.75 & 157 & 1.1 & 7.1 \\
Entangled UHMWPE Grade A & 1.95 & 102 & 1.2 & 1.9 \\
Entangled UHMWPE Grade B & 2.30 & 165 & 1.1 & 6.0 \\
Solution spun Fiber & 3.50 & 135 & 2.2 & 0.3 \\
\hline
\end{tabular}

Figure 3. Orientation of crystalline planes of polyethylene with increasing tensile modulus or corresponding draw ratio (Figure 2). Polyethylene chains in the unconstrained state pack in the orthorhombic unit cell. The crystalline planes are randomly oriented in the nascent powders of the entangled and disentangled polymers. For the isotropic distribution of the crystallites the intensity ratio between the 200/110 reflection is 0.3 , whereas the intensity ratio increases with uniaxial deformation. For the same draw ratio of nearly 180 times crystal plane orientation (CPO) is significantly higher in the disentangled sample (exceeding 7.0) compared to the solution-spun fibers having crystal plane orientation of 0.3.

made use of Dyneema SK75 grade over the SK78 grade because of its higher modulus and tensile strength. For comparison on mechanical properties between the two commercially available fibers, Dyneema SK75 and SK78, and the data reported in literature on the solution-spun fibers at laboratory scale see the Supporting Information and refs 4, 7, 9, 10, 12, 32, and 34. The modulus and strength of the commercially available solutionspun fiber, SK75, are the highest among the solution-spun fibers and are in the region of 135 and $3.5 \mathrm{GPa}$, respectively. On comparing Figure 2 with the modulus and strength of the fibers, it is apparent that the tensile strength of the solution-spun fiber matches with the disentangled tape obtained via the solvent-free route. However, modulus of both tapes, disentangled as well as entangled, outperforms that of the solution-spun fiber. To understand cause for the higher modulus of the tape over the fiber, X-ray diffraction and solid-state NMR studies are performed.

On crystallization, from solution or melt, linear polyethylene chains pack in the orthorhombic unit cell. Depending on the distribution of the crystalline units in the three-dimensional plane, intensities of the crystal reflections vary. For example, in isotropic distributed crystalline units, the intensity ratio between the two most intense reflections 200/110 determined theoretically is $0.3 .^{36}$ The intensity ratio changes with the preferred orientation of the crystallites and thus could be a signature to provide information on crystal plane orientation (CPO). The three different possibilities that can arise and would cause changes in the intensity ratio are depicted in Figure 3. From such studies it could be concluded that the polymer prior to compression, as obtained from the reactor, shows isotropic distribution of crystalline planes; thus, the intensity ratio of 200/110 reflection matches the theoretical value of 0.3 . For the $\mathrm{X}$-ray beam size of $100 \mu \mathrm{m}$, the commercially available fiber spun from solution shows preferred orientation of the crystallites along the chain axis, but random distribution of the crystallite planes ( $h k 0)$, thus resulting in the intensity ratio of 200/110 reflections 0.3 . On the contrary, tapes as obtained from the disentangled and entangled polyethylenes for the same X-ray beam size (in reflection mode) show increase in the intensity ratio of $200 / 110$ reflections that increases with draw ratio and the corresponding tensile modulus (Figure 2), thus suggesting preferred orientation of the crystalline planes in the tapes as summarized in Figure 3. X-ray diffraction patterns are recorded on the samples mounted on a goniometer having draw direction perpendicular to the rotation axis. To avoid any preferred orientation, the sample is continuously rotated at $15 \mathrm{cpm}$. For details of the experiments see the Experimental Methods section and the Supporting Information. From such studies it is apparent that the intensity ratio between the 200/110 reflection changes with increasing tensile strength of the tapes. The increase in the intensity ratio is indicative of the development of crystal plane orientation (CPO) along with chain orientation on deformation. Thus, no preferred planar orientation is observed in the solution-spun samples, while the chains are oriented along the draw direction.

The high crystal plane orientation suggests high overlapping of the crystalline planes, thus leading to higher van der Waals interactions between the neighboring chains. This unique enhancement in the van der Waals interactions between the neighboring chains in the solid state processed polyethylene is most likely to be a cause for high modulus in the polyethylene tapes compared to the solution-spun fiber where orientation is achieved only along the c-axis. In Figure 3, this is shown with examples of two different commercially available entangled polyethylenes - for practical uses hereafter called A and B. Grade A could be processed to a tensile modulus and strength of 
a) ${ }^{13} \mathrm{C}$ CP/MAS Spectra of drawn UHMWPES

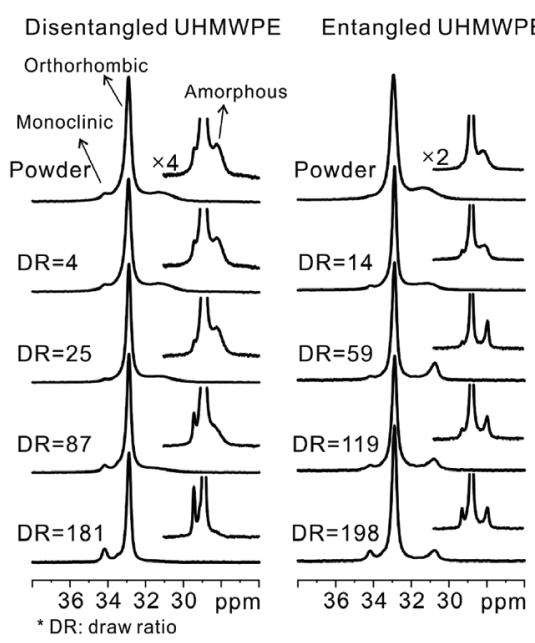

b) Crystallinity variation in drawn UHMWPEs

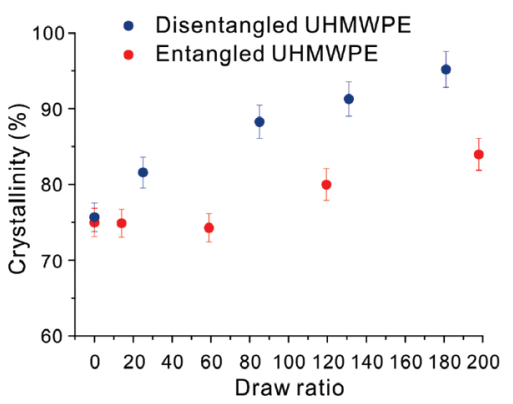

c) ${ }^{13} \mathrm{C} \mathrm{SP} / \mathrm{MAS}$ Spectra of drawn UHMWPES
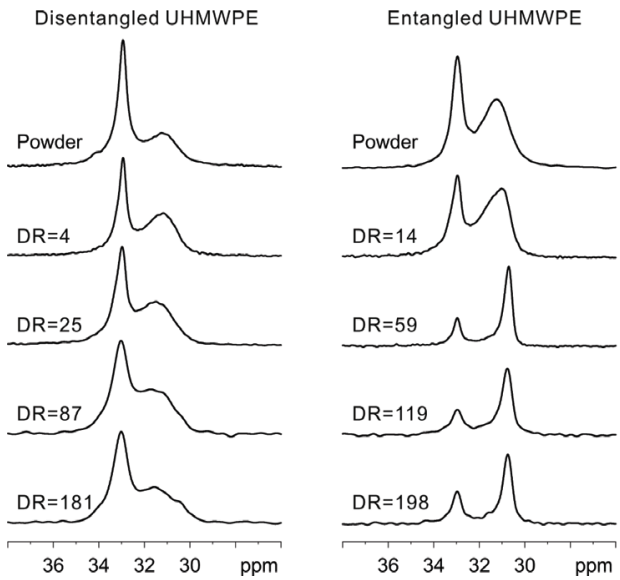

Figure 4. Changes in the amorphous state of entangled and disentangled UHMWPE with increasing draw (or stretching) ratio recorded at $50{ }^{\circ} \mathrm{C}$. (a) ${ }^{13} \mathrm{C} \mathrm{CP} / \mathrm{MAS}$ spectra of UHMWPE tapes drawn at different draw ratios. For clarity of the weak amorphous peak spectra have been magnified for four times. From the figure it is apparent that in the disentangled sample amorphous peak decreases whereas the peak sharpen and becomes more evident in the entangled sample. (b) Plot of increase in crystallinity, as measured by NMR spectra from (a), with draw ratio. Differences in the crystallinity with draw ratio. (c) ${ }^{13} \mathrm{C} \mathrm{SP} / \mathrm{MAS}$ spectra recorded at different draw ratios. The single pulse (SP) spectra are acquired after a short delay time of $3 \mathrm{~s}$, where changes in the amorphous chain segments are followed by suppressing relaxation arising due to the crystalline chain segments having relatively very long relaxation time.

respectively 110 and $1.9 \mathrm{GPa}$. These mechanical properties are lower than that of tapes processed from disentangled polyethylenes; viz. tensile modulus and strength of 165 and $3.8 \mathrm{GPa}$, respectively. The crystalline order along the $c$-axis is similar to that of tapes processed from disentangled polyethylene but remarkably higher than that of solution-spun filaments as shown in the table in Figure 3. The observed planar orientation is significantly lower than that of the disentangled polyethylene tape sample, and here it should be noted that this is in correspondence to the lower mechanical properties. Grade B could be processed to a tensile modulus and strength of 165 and $2.2 \mathrm{GPa}$, respectively. The tensile modulus is similar to that of the disentangled polyethylene and interestingly is encompassed with an almost identical intensity ratio for the $200 / 110$ reflections.

However, the tensile strength for the tape processed from grade B is not on the same level as that of the tape processed from disentangled polyethylene or the commercially available solution-spun polyethylene fibers. The cause of this difference in tensile strength can be associated with the morphological aspects and orientation of the amorphous region that cannot be easily investigated by X-ray diffraction. For the purpose we performed solid-state NMR studies.

In semicrystalline polymers, both crystalline and amorphous components have a strong influence on the mechanical properties. For an example, because of the length of polymer chain, a chain residing within a crystal may traverse to the other passing through the amorphous region. The content of amorphous components and the extent to which the amorphous chain segments are tight or loose are critical for the mechanical properties. To probe the amorphous components and further correlate them to the mechanical properties, solid-state NMR studies have been performed on several UHMWPEs at different draw ratios. 
Figure 4a shows ${ }^{13} \mathrm{C}$ NMR spectra of the disentangled and the entangled UHMWPEs acquired by using ${ }^{1} \mathrm{H} \rightarrow{ }^{13} \mathrm{C}$ crosspolarization combined with magic angle spinning (CP/MAS). To recall, in such a spectrum, the sharp peak observed at $\sim 33$ ppm is assigned to all-trans conformations in the orthorhombic crystals, while the signal at $\sim 31 \mathrm{ppm}$ can be assigned to the gauche conformations present in amorphous regions. From the magnified spectra in Figure 4a, it is clear that the amorphous peaks of the disentangled and entangled samples develop differently with increasing draw ratio. For the entangled sample, with increasing the draw ratio the amorphous peaks do not show a remarkable decrease in the intensity but rather demonstrate a clear narrowing of the peak width, indicating the high mobility of the segments developing during the deformation, a point that will be discussed later. For the disentangle samples, however, the amorphous peaks gradually disappear with the increasing draw ratio. At high draw ratios (e.g., draw ratio $=181$ ), the amorphous peak nearly disappears. The disappearance of the amorphous peak indicates significant conformer transformations from gauche to the all-trans occurring during the drawing process, which in turn results in the increase in the sample crystallinity. Figure $4 \mathrm{~b}$ depicts the variation of the sample crystallinity with increasing draw ratio, where the crystallinity of the disentangled samples increases from $\sim 75 \%$ to $\sim 95 \%$ during deformation of the compressed powder to the maximum draw ratio of 181 . For the entangled samples the crystallinity keeps almost unchanged in the wide draw ratio range from 0 to 119 . At the maximum draw ratio of 198 , crystallinity of the sample slightly increases to $\sim 84 \%$ that is still far below the crystallinity of the disentangled sample at the same range of draw ratio.

In the spectra shown in Figure 4a accompanied by the disappearance of the amorphous signal, a small peak at $\sim 34.2$ $\mathrm{ppm}$ increases gradually. In the literature, ${ }^{37}$ this peak has been attributed to all-trans conformations in the monoclinic phase that arises due to shearing of the orthorhombic crystals during solidstate deformation. The increase in intensity of the monoclinic peak, where chain segments are also in the all-trans conformation, indicates increase in the amount of sheared crystals on solidstate deformation.

The extent to which the amorphous chain segments are tight or loose in the PE samples is probed by monitoring the position and width of the amorphous signal in the ${ }^{13} \mathrm{C}$ spectrum. The position and width of the amorphous signal depend on the conformational statistics and the accessibility of the conformational space to dynamic processes present in the amorphous regions and thus can be indicative of the tightness of the chain segments. Figure $4 \mathrm{c}$ shows the single pulse (SP) excitation spectra of the drawn disentangled and entangled samples under magic angle spinning (SP/MAS) acquired by using a short recycle delay time of $3 \mathrm{~s}$. In these spectra, the crystalline signal is considerably suppressed because of the very long relaxation time of the ${ }^{13} \mathrm{C}$ spins in the crystalline regions (usually more than $1000 \mathrm{~s}$ ). The crystalline signal in the spectra arises mainly due to the exchange process of the chain segments from amorphous to crystalline region in the semicrystalline polymer. The mechanism has been explained in detail elsewhere. ${ }^{27}$ Adjacent to the crystalline peak, the broad amorphous peak in the spectra of the nascent powder ( $\sim 31.2$ and $1.68 \mathrm{ppm}$ peak width) is different from the amorphous signal of melt crystallized $\mathrm{PE}^{27}(\sim 30.5$ and $0.58 \mathrm{ppm}$ peak width), reflecting restriction in mobility in the nascent disentangled sample to adopt different conformations. With increasing draw ratio the amorphous peak broadens further, that

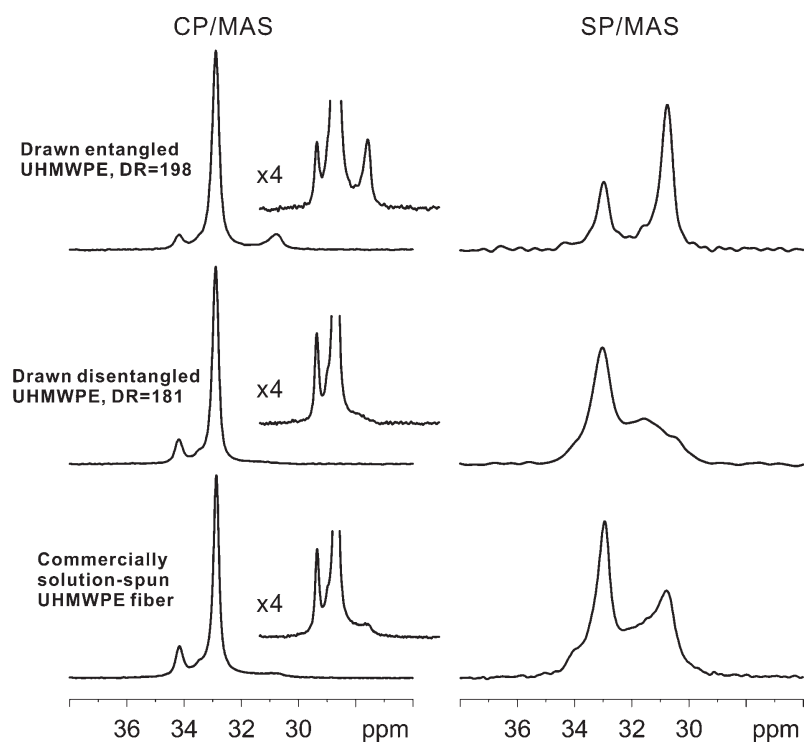

Figure 5. NMR spectra recorded for the three fully drawn samples of UHMWPE, via solid-state solvent free processing routes and solution-spinning. The spectra for SP/MAS is recorded after $3 \mathrm{~s}$ delay time at $50{ }^{\circ} \mathrm{C}$. From the figures it is evident that the fully drawn disentangled sample contains minimum amount of the mobile amorphous component.

is indicative of the restriction in chain conformation imposed by stretching of chains during solid-state deformation. On combining the CP/MAS and the SP/MAS spectrum, it can be concluded that with increasing draw ratio, in the solid state, the amorphous component in the nascent disentangled polyethylene tend to decrease and adopts more restricted mobility.

Figure 5 compares the NMR spectrum recorded under same conditions in the maximum solid-state deformed entangled, disentangled, and solution-spun UHMWPE. In the CP/MAS spectrum the solid-state deformed entangled UHMWPE shows the presence of mobile amorphous component $(\sim 30.5 \mathrm{ppm}$ with a peak width of $\sim 1.0 \mathrm{ppm}$ ). For the entangled sample SP/MAS shows comparatively low intensity peak at $\sim 33.0 \mathrm{ppm}$. This suggests considerable restriction in translation of chain segments from amorphous to crystalline region. The restriction in the translation may be attributed to the entropic barrier that arises due to considerable conformational differences in the mobile amorphous and the rigid crystalline components, an observation reported earlier on UHMWPE. ${ }^{27}$ In the disentangled UHMWPE, due to decreasing conformational differences between the amorphous and the crystalline regions, with increasing draw ratio the entropic barrier at high deformation lowers thus peak intensity at the orthorhombic position is more intense. The SP/MAS NMR spectrum of the disentangled solid-state drawn tape shows more similarity with the solution-spun fibers than with the entangled solid-state drawn tapes. However, unlike the disentangled solidstate drawn sample the solution-spun sample shows the presence of some mobile component (having gauche conformers) together with the restricted amorphous component.

A comparison of CP/MAS spectra (Figure 5) of the three fully drawn samples shows prominent presence of mobile amorphous component only in the entangled sample. Some traces of the mobile component are also present in the solution-spun sample. However, even at high magnification, no presence of mobile amorphous component is found in the disentangled sample. On 


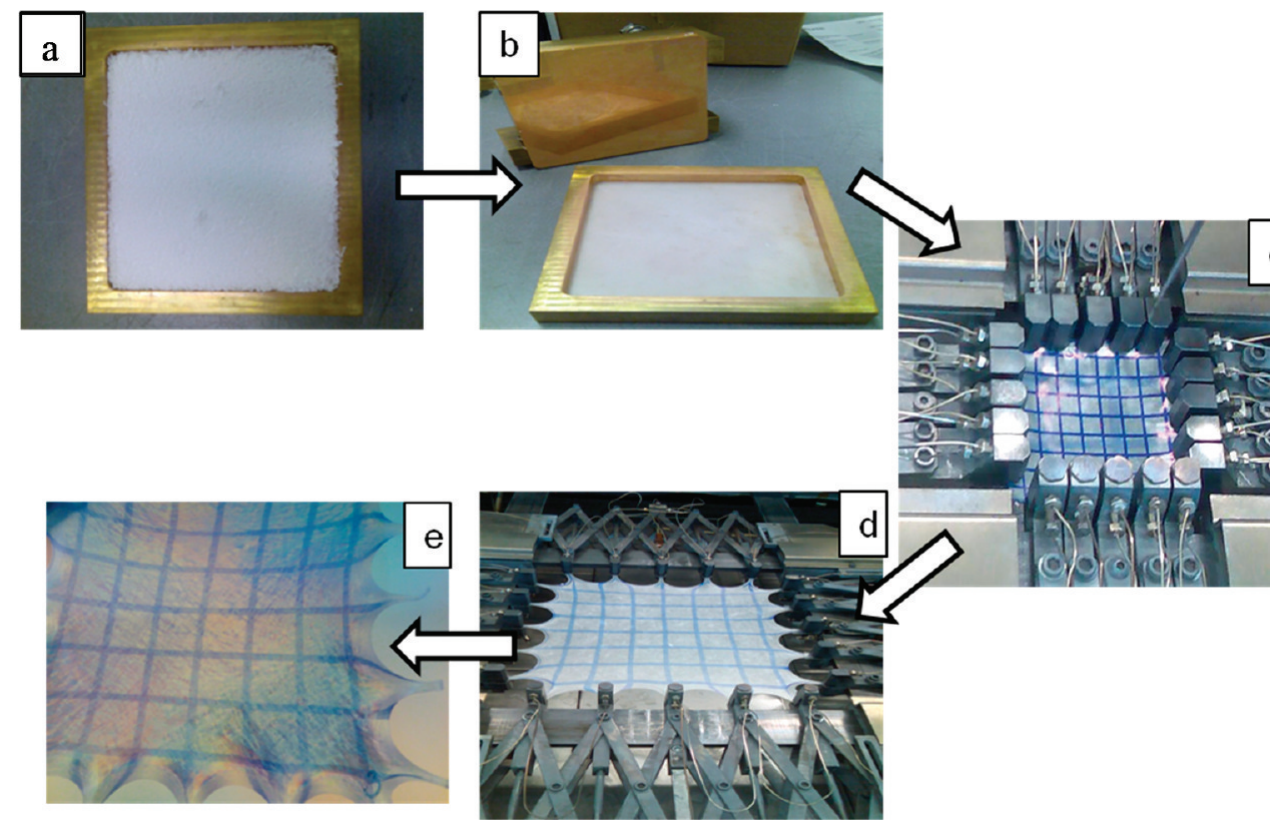

Figure 6. Biaxial stretching of the compressed disentangled UHMWPE. (a) Filled disentangled UHMWPE powder in mold, prior to compression at $120^{\circ} \mathrm{C}$. The compacted film obtained after compression is shown in the mold in (b). After rolling process at $120^{\circ} \mathrm{C}$, the $225 \mu \mathrm{m}$ thick compressed film is mounted on a biaxial stretching machine (c). The sample is stretched biaxially at $130^{\circ} \mathrm{C}$. Maximum draw ratio achieved by one shot stretching process is $10 \times 10$ times where the sample thickness reduces from $225 \mu \mathrm{m}$ to nearly $8 \mu \mathrm{m}(\mathrm{d})$.

summarizing the CP/MAS and SP/MAS spectra, it could be stated that the mobility of the amorphous component in the solution-spun sample is closer to the solid-state drawn disentangled sample. This observation can be linked to the difference in the tensile strengths of the three samples, which is highest for the solid-state drawn disentangled UHMWPE and lowest for the entangled UHMWPE.

The ease in uniaxial deformation of the disentangled nascent sample due to wide processing window (more than $15{ }^{\circ} \mathrm{C}$ compared to $2 / 3{ }^{\circ} \mathrm{C}$ of the entangled sample) in the solid state provides a unique opportunity to explore the possibility of biaxial stretching. In general, low molar mass polyethylene films are produced by blow molding. However, due to poor tensile strength, the application of such films is limited to commodity applications. In order to fulfill some of the requirements for more demanding applications such as membranes, waterproof breathable articles, (di)electric properties, coating for elastomers, etc., the high abrasion resistance combined with outstanding modulus and tensile strength of the UHMWPE films are desired. Similar to the challenges that one encounters in processing of the uniaxial films, at present the commercially available biaxial porous films of UHMWPE are produced using more than $95 \mathrm{wt} \%$ of the solvent. The method applied for the porous film production makes use of solution spinning route for fibers where instead of spinneret a slit die is used for making of tapes. Following the controlled evaporation of the solvent the gel-spun tapes are converted into porous films for membrane applications.

The disentangled polyethylene provides an unprecedented opportunity to process the intractable polymer in biaxial direction in the solid state. Following the route described in a patent application, ${ }^{38}$ biaxially stretched nonporous films of nearly $8 \mu \mathrm{m}$ thickness having isotropic tensile strength approaching $0.7 \mathrm{GPa}$ could be obtained for the first time (see Figure 6). The biaxial drawn film when viewed between cross-polars show continuous variation in color from blue to red along the azimuthal angle. The variation in color is indicative of the addition and subtraction of the wavelength in the polarized light with the retardation thickness of the film, following the first order in the MichelleLevy chart. The continuous variation in color arises due to isotropic distribution of the stretched chains having average molar mass of 8 million $\mathrm{g} / \mathrm{mol}$ and polydisperisty of nearly 3.0. Estimated isotropic tensile strength of the film is $0.7 \mathrm{GPa}$. Biaxial stretching, in the solid state, is the unique property of the disentangled UHMWPE and cannot be obtained, without using solvent, in the entangled polymer. Further details on biaxial drawn films will be provided in a separate publication.

The controlled synthesis reported in this publication also provides a possibility to add nanofillers during polymerization such as hydroxylapatite, carbon nanotubes, etc., that could be also compressed and drawn uniaxially as well as biaxially. Such composites provide possibility to make high-modulus, highstrength membranes or tapes that could be used for biocompatible applications in the case of hydroxylapatite or thermal exchange process in the uniaxial or isotropic direction in the case of carbon nanotubes. ${ }^{39}$

\section{CONCLUSIONS}

From the studies reported in this Communication the following conclusions can be drawn:

(a) With the help of solid-state NMR, following the chain diffusion process between the amorphous and the crystalline regions, distinction between the topological constraints of the samples synthesized using conventional $\mathrm{Z}-\mathrm{N}$ catalyst and the single-site catalytic system could be made.

(b) Compared to the entangled polyethylene, that can be drawn within a narrow temperature window of $2-3{ }^{\circ} \mathrm{C}$, 
the disentangled polyethylene can be drawn in a broad temperature window of $20{ }^{\circ} \mathrm{C}$ below the equilibrium melting temperature, thus making it easier to process.

(c) The deformation of the disentangled polyethylene, below the melting temperature, shows continuous increase in crystallinity compared to the entangled polyethylene where the crystallinity drops at the lower draw ratios and attains relatively low values. The cause of lower crystallinity in the entangled polyethylene is the partial melting process that arises during deformation near the melting point.

(d) The tensile modulus of the solid-state drawn tapes (>165 GPa for disentangled and $\sim 140 \mathrm{GPa}$ for entangled) is higher than that of the solution-spun fibers (maximum $130 \mathrm{GPa}$ ) because of the higher crystal plane orientation that could be achieved in the solid-state processed polymers, though crystallinity of the solutionspun fiber is comparable with the disentangled solid-state processed tapes.

(e) The higher tensile strength $(>3.8 \mathrm{GPa})$ in the disentangled polyethylene compared to the entangled one $(\sim 2.3 \mathrm{GPa})$ is attributed to the narrower molar mass distribution of the disentangled polyethylene and the higher segmental mobility present in the amorphous region.

(f) Unlike the entangled polyethylene that could not be drawn biaxially without using a solvent, the disentangled polymer could be drawn in its solid state, free of solvent.

The salient finding in the reported study is that in the semicrystalline polymer uniaxial and biaxial deformation are strongly dependent on the topological constraints residing in the amorphous region. Thus, it is essential to control the topological constraints either via controlled synthesis or dis solution process. To our knowledge the above summarized results and the properties on the disentangled polyethylene, obtained via controlled synthesis, are unprecedented and have the potential to open a new fundamental understanding to bridge polymer morphology with the resultant mechanical properties.

\section{ASSOCIATED CONTENT}

Supporting Information. Experimental details. This material is available free of charge via the Internet at http://pubs.acs. org.

\section{AUTHOR INFORMATION}

\section{Corresponding Author}

${ }^{*}$ E-mail: s.rastogi@lboro.ac.uk.

\section{ACKNOWLEDGMENT}

The authors thank Teijin Aramid B.V, Netherlands, for the financial support and for providing the commercial entangled samples used in this publication.

\section{REFERENCES}

(1) de Gennes, P. G. J. Chem. Phys. 1971, 55, 572-579.

(2) Likhtman, A. E.; McLeish, T. C. B. Macromolecules 2002, $35,6332-6343$.
(3) Vega, J. F.; Rastogi, S.; Peters, G. W. M.; Meijer, H. E. H. J. Rheol. 2004, 3, 663-678.

(4) Smith, P.; Lemstra, P. J.; Pijpers, J. P. L. J. Polym. Sci., Polym. Phys. 1982, 20, 2229-2241.

(5) Keller, A. Philos. Mag. 1957, 2, 1171-1175.

(6) Rastogi, S.; Spoelstra, A. B.; Goossens, J. G. P.; Lemstra, P. J. Macromolecules 1997, 30, 7880-7889.

(7) Bastiaansen, C. W. M. J. Polym. Sci., Part B: Polym. Phys. 1990, $28,1475-1482$.

(8) Smith, P.; Lemstra, P. J. US Patent 4344908, 1982.

(9) Bastiaansen, C. W. M. Oriented structures based on flexible polymers. PhD Thesis, Eindhoven University of Technology, 1991.

(10) Lemstra, P. J.; Bastiaansen, C. W. M.; Rastogi, S. Structure Formation in Polymeric Fibres; Hanser Publisher: Munich, 2000.

(11) Rotzinger, B. P.; Chanzy, H. D.; Smith, P. Polymer 1989, 30, 1814-1819.

(12) Chanzy, H. D.; Rotzinger, B. P.; Smith, P. US Patent 4769433, 1988.

(13) Rastogi, S.; Sharma, K.; Duchateau, R.; Gruter, G. J. M.; Lippits, D. R. US Patent 0142521, 2006.

(14) Rastogi, S.; Lippits, D. R.; Höhne, G. W. H.; Mezari, B.; Magusin, P. C. M. J. Phys.: Condens. Matter 2007, 19, 205122.

(15) Lippits, D. R.; Rastogi, S.; Höhne, G. W. H.; Mezari, B.; Magusin, P. C. M. Macromolecules 2007, 40, 1004-1010.

(16) Lippits, D. R.; Rastogi, S.; Höhne, G. W. H. Phys. Rev. Lett. 2006, 96, 218303.

(17) Lippits, D. R.; Rastogi, S.; Talebi, S.; Bailly, C. Macromolecules 2006, 39, 8882-8885.

(18) Rastogi, S.; Lippits, D. R.; Peters, G. W. M.; Graf, R.; Yefeng, Y.; Spiess, H. W. Nature Mater. 2005, 4, 635-641.

(19) Peacock, A. J. Handbook of Polyethylene; Marcel Dekker: New York, 2000.

(20) Brintzinger, H. H.; Fischer, D.; Mülhaupt, R.; Rieger, B.; Waymouth, R. M. Angew. Chem., Int. Ed. 1995, 34, 1143-1170.

(21) For sake of simplicity, we will indicate as "heterogeneous synthesis route" the process involving the use of a heterogeneous catalytic system and as "homogeneous synthesis route" the one involving the use of an homogeneous catalytic system, even if, in the latter case, the system will become biphasic as soon as the polymer starts to precipitate out of the solution.

(22) Mitani, M.; Saito, J.; Ishii, S.; Nakayama, Y.; Makio, H.; Matsukawa, N.; Matsui, S.; Mohri, J.; Furuyama, R.; Terao, H.; Bando, H.; Tanaka, H.; Fujita, T. Chem. Rev. 2004, 4, 137-158.

(23) Saito, J.; Mitani, M.; Mohri, J.; Yoshida, Y.; Matsui, S.; Ishii, S.; Kojoh, S.; Kashiwa, N.; Fujita, T. Angew. Chem., Int. Ed. 2001, 40, 2918-2920.

(24) Talebi, S.; Duchateau, R.; Rastogi, S.; Kaschta, J.; Peters, G. W. M.; Lemstra, P. J. Macromolecules 2010, 43, 2780-2788.

(25) Klimke, K. Macromol. Chem. Phys. 2006, 207, 382-395.

(26) Yao, Y.; Graf, R.; Spiess, H. W.; Rastogi, S. Macromol. Rapid Commun. 2009, 30, 1123-1127.

(27) Yao, Y.; Graf, R.; Spiess, H. W.; Rastogi, S. Macromolecules 2008, 41, 2514-2519.

(28) Kanamoto, T.; Sherman, E. S.; Porter, R. S. Polym. J. 1979, 11, 497-502.

(29) Zachariades, A. E.; Watts, M. P. C.; Porter, R. S. Polym. Eng. Sci. 1980, 20, 555-561.

(30) Kanamoto, T.; Tsuruta, A.; Tanaka, K.; Takeda, M. Polym. J. 1984, 16, 75-79.

(31) Kanamoto, T.; Ohama, T.; Tanaka, K.; Takeda, M.; Porter, R. S. Polymer 1987, 28, 1517-1520.

(32) Smith, P.; Chanzy, H. D.; Rotzinger, B. J. Mater. Sci. 1987, 22, 523-531.

(33) De Weijer, A. P.; Peters, M. W. M. G.; van de Hee, H.; Rastogi, S.; Wang, B. European patent application EP 2014445A1, 2009.

(34) Lemstra, P. J.; Smith, P. Br. Polym. J. 1980, 212-214.

(35) Pandey, A.; Champouret, Y.; Rastogi, S. Macromolecules 201110.1021 
(36) Bunn, C. W. Trans. Faraday Soc. 1939, 35, 482-491.

(37) Benn, R.; Fink, G.; Herrman, W.; Muller, T. 1992, 13, $321-327$.

(38) Van der Eem, J.; Bos, J.; de Weijer, A. P.; Rastogi, S.; Elderman, G. WO 2010/079174 A2, 2010.

(39) Rastogi, S.; Ronca, S. WO 2010/079173 A1, 2010. 